TITLE:

\title{
Cross sections for ionization of uracil by MeV-energy-proton impact
}

$\operatorname{AUTHOR}(S)$ :

Itoh, A.; Iriki, Y.; Imai, M.; Champion, C.; Rivarola, R. D.

\section{CITATION:}

Itoh, A.... [et al]. Cross sections for ionization of uracil by MeV-energyproton impact. Physical Review A 2013, 88(5): 052711.

ISSUE DATE:

2013-11-27

URL:

http://hdl.handle.net/2433/180088

RIGHT:

(C) 2013 American Physical Society 


\title{
Cross sections for ionization of uracil by MeV-energy-proton impact
}

\author{
A. Itoh, ${ }^{1,}{ }^{*}$ Y. Iriki, ${ }^{1}$ M. Imai, ${ }^{1}$ C. Champion, ${ }^{2}$ and R. D. Rivarola ${ }^{3}$ \\ ${ }^{1}$ Department of Nuclear Engineering, Kyoto University, Kyoto 615-8540, Japan \\ ${ }^{2}$ Université Bordeaux 1, CNRS/IN2P3, Centre d'Études Nucléaires de Bordeaux-Gradignan (CENBG), France \\ ${ }^{3}$ Instituto de Física Rosario, CONICET and Universidad Nacional de Rosario, Argentina \\ (Received 1 October 2013; revised manuscript received 1 November 2013; published 27 November 2013)
}

\begin{abstract}
Absolute double differential cross sections (DDCS) have been measured for the ionization of gas-phase uracil $\left(\mathrm{C}_{4} \mathrm{H}_{4} \mathrm{~N}_{2} \mathrm{O}_{2}\right)$ by $0.5,1.0$, and $2.0 \mathrm{MeV}$ proton impacts. Measurements were performed by secondary electron spectroscopy using a $45^{\circ}$ parallel plate electrostatic spectrometer over an energy range of $1.0-1000 \mathrm{eV}$ at emission angles from $15^{\circ}$ to $165^{\circ}$. Theoretical calculation of ionization cross sections has also been carried out by using the first-order Born approximation with correct boundary conditions. Single differential and total ionization cross sections deduced from DDCS revealed fairly good agreement between experimental and theoretical values at all the emission angles investigated.
\end{abstract}

DOI: 10.1103/PhysRevA.88.052711

PACS number(s): $34.50 . \mathrm{Gb}$

\section{INTRODUCTION}

The ion-impact ionization of atoms and molecules has been studied extensively in the past [1-8]. Experimental studies range widely from traditional measurements of electron emission cross sections, as reviewed in [1-4], to multiple differential cross-section measurements [5-8]. Because of a wide range of application fields such as thermonuclear fusion, biology, and medical therapy, ionization cross sections of water and hydrocarbon molecules have been investigated for a long time [9-19]. More recently, rather complicated biological molecules like nucleobases have received increasing attention. Tabet et al. [20] measured proton-impact total ionization cross sections (TICS) of nucleobases such as adenine and uracil by means of time-of-flight fragment ion measurements at proton energies below $150 \mathrm{keV}$. Moretto-Capelle and Le Padellec [21] measured energy distributions of secondary electrons emitted from uracil by protons with energies below $100 \mathrm{keV}$. As for high-energy heavy ion impacts, Agnihortri et al. have measured the total ionization cross sections of uracil bombarded by highly charged $\mathrm{C}^{q+}$ and $\mathrm{O}^{q+}$ ions $(q \geqslant 4)$ at energies of $0.1-78 \mathrm{MeV}$ [22]. Theoretical studies on such nucleobases have also been performed extensively by using various theoretical models. Abbas et al. [23] and Lekadir et al. [24] obtained TICS of nucleobases by proton and alpha particles by using a classical trajectory Monte Carlo method combined with the classical over barrier approximation (CTMC-COB). Champion et al. obtained cross sections by the first-order Born approximation [25]. They presented not only TICS but also double- and single-differential cross sections for electron emission from nucleobase molecules. These cross sections are abbreviated in the following as DDCS and SDCS, respectively.

It is known that secondary electrons play an important role in radiation-induced biological effects [26-28]. Indeed, in a collision with fast charged particles, a large number of electrons may be produced in a Bragg peak region where incident ions lose most of their kinetic energies. These electrons may induce ionization and fragmentation of neighboring

*itoh@nucleng.kyoto-u.ac.jp molecules when the kinetic energies of impacting electrons are sufficiently high. Also, low-energy electrons of below $50 \mathrm{eV}$, which are produced predominantly in fast ion-atom collisions, are known to induce damages to DNA or RNA bases via dissociative electron attachment [26,27].

To achieve precise understanding and predictions about the role of secondary electrons, it is essentially important to know the energy and angular distributions of these electrons. However, reliable experimental cross sections for biological molecules are still largely lacking at present.

Upon this motivation we started recent measurements of ionization cross sections of nucleobase molecules by means of electron spectroscopy and reported a series of $\mathrm{MeV}$ energy proton-impact data for adenine molecules $\left(\mathrm{C}_{5} \mathrm{H}_{5} \mathrm{~N}_{5}\right)$ in two papers [29] which will be referred to as Ref. I hereafter.

In this work, we extend measurements to an RNA base uracil target $\left(\mathrm{C}_{4} \mathrm{H}_{4} \mathrm{~N}_{2} \mathrm{O}_{2}\right)$ by $0.5-2.0 \mathrm{MeV}$ proton impact. Quantum-mechanical calculations are also performed in an impact energy range of $0.05-10 \mathrm{MeV}$ by using the first-order Born approximation with correct boundary conditions, cited as CB1 hereafter. Experimental and theoretical results for differential and total cross sections are examined in detail. A comparison is also made with a simple analytical formula proposed by Stolterfoht et al. [4] using the model of classical Rutherford knock-on collisions.

\section{EXPERIMENT}

The experimental apparatus and procedure to obtain cross sections are described in detail in our previous paper of Ref. I, and only an essential outline is described below. A beam of protons with energies $0.5,1.0$, and $2.0 \mathrm{MeV}$ was produced by a Van de Graff accelerator of the Quantum Science and Engineering Centre heavy ion accelerator facility of Kyoto University. The beam was collimated to a size of about $1 \times 3 \mathrm{~mm}^{2}$ by a magnetic lens and charge-purified by a magnetic charge selector before entering a collision chamber. After collision with a gaseous uracil target, the beam was collected by a Faraday cup. A typical beam current was about $50 \mathrm{nA}$. Simultaneously, we also detected projectile particles scattered into forward angles of $2.5^{\circ}$ to determine the effective target thickness of uracil. An effusive molecular 
beam target of uracil was produced from crystalline uracil powder of $99 \%$ purity contained in a 5.3-cm-long stainless steel oven placed inside a copper container equipped with two cylinder-shaped heaters. The measurements of secondary electrons were carried out at an oven temperature of $473 \mathrm{~K}$. The molecular beam of uracil was ejected through an outlet aperture of $1 \mathrm{~mm}$ in diameter placed at the top of the oven and was trapped by a water-cooled copper plate placed at $100 \mathrm{~mm}$ above the proton beam line. The position of the outlet aperture was $3 \mathrm{~mm}$ below the beam line. A base pressure of the collision chamber was kept below $2 \times 10^{-7}$ Torr during the experiment. Ejected electrons were analyzed by a $45^{\circ}$ parallel plate electrostatic spectrometer mounted on a turntable controlled by a pulse motor. Electrons were detected by a channel electron multiplier (CEM). The residual or Earth's magnetic field inside the collision chamber was reduced to less than a few $\mathrm{mG}$ by double permalloy magnetic shields. Measurements were carried out in an electron energy $(\epsilon)$ range of $1.0-1000 \mathrm{eV}$ at emission angles $(\theta)$ from $15^{\circ}$ to $165^{\circ}$ at $15^{\circ}$ intervals with respect to the direction of the $\mathrm{H}^{+}$beam. The energy resolution $\Delta \epsilon / \epsilon$ of the electron spectrometer was $8 \%$ at FWHM. To collect efficiently low-energy electrons of a few $\mathrm{eV}$, a positive bias of $40 \mathrm{~V}$ was applied as an extraction voltage into the spectrometer.

By using DDCS $\sigma(\epsilon, \theta)$, the cross sections SDCS and TICS are obtained, respectively, as

$$
\sigma(\epsilon)=2 \pi \int_{0}^{\pi} \sigma(\epsilon, \theta) \sin \theta d \theta, \quad \sigma_{t}=\int_{0}^{\epsilon_{m}} \sigma(\epsilon) d \epsilon,
$$

with $\epsilon_{m} \simeq 1000 \mathrm{eV}$.

\section{THEORY}

\section{A. Quantum-mechanical calculation}

In the present work, the biomolecule is described via its molecular orbitals by employing the quantum chemical GAUSSIAN 03 program. Briefly, let us note that the target wave functions were computed at the Hartree-Fock level optimized at the MP2/6-31G(d) computational level, i.e., by including correlation calculations at the second order of perturbation theory MP2 and by using GAUSSIAN-type orbitals added to a double-zeta valence shell and polarization orbitals on nonhydrogen atoms. Total-energy calculations were then performed in the gas phase with the GAUSSIAN 09 software at the RHF/3-21G level of theory. Furthermore, the ionization potentials also calculated at the RHF/3-21G level have shown a very good agreement with the experiments [30]. The effective number of electrons relative to any atomic component of each molecular orbital was derived from a standard Mulliken population analysis [31].

Under these conditions, the target molecule ionization cross sections, whatever their degree of differentiation, were seen as a linear combination of atomic orbital (LCAO) cross sections corresponding to the different component of the investigated target $(\mathrm{H}, \mathrm{C}, \mathrm{N}, \mathrm{O})$ weighted by the effective occupation electron number, namely

$$
\sigma=\sum_{j=1}^{N} \sigma_{j}=\sum_{j=1}^{N} \sum_{i}^{N_{j}} \xi_{j, i} \sigma_{a t, i}
$$

where $N$ refers to the number of molecular orbitals of the impacted biomolecule ( $N=29$ for uracil) while $N_{j}$ denotes the total number of atomic components of the $j$ th molecular orbital, $\sigma_{a t, i}$ the corresponding atomic orbital cross sections, and $\xi_{j, i}$ the effective number of electrons involved in the present LCAO description. For more details, we refer the reader to our previous work [25,31].

In the CB1 model, the initial and final wave functions are chosen as (in atomic units)

$$
\varphi_{\alpha}^{+}=\frac{\exp \left(i \boldsymbol{K}_{\alpha} \cdot \boldsymbol{R}\right)}{(2 \pi)^{3 / 2}} \phi_{\alpha}(\boldsymbol{x}) \exp \left[-i \frac{Z_{p}}{v} \ln (v R-\boldsymbol{v} \cdot \boldsymbol{R})\right]
$$

and

$$
\begin{aligned}
\varphi_{\beta}^{-}= & \frac{\exp \left(i \boldsymbol{K}_{\beta} \cdot \boldsymbol{R}\right)}{(2 \pi)^{3 / 2}} \phi_{\beta}(\boldsymbol{x}) N^{*}\left(Z_{T}^{*} / k\right) \\
& \times{ }_{1} F_{1}\left(-i Z_{T}^{*} / k ; 1 ;-i k x-i \boldsymbol{k} \cdot \boldsymbol{x}\right) \\
& \times \exp \left[+i \frac{Z_{p}}{v} \ln (v R+\boldsymbol{v} \cdot \boldsymbol{R})\right],
\end{aligned}
$$

where the vectors $\boldsymbol{x}$ and $\boldsymbol{R}$ give the position of the active electron and the projectile with respect to the center of mass of the target, respectively, $\boldsymbol{k}$ denotes the momentum of the ejected electron seen from the target, and $\boldsymbol{K}_{\alpha}$ and $\boldsymbol{K}_{\beta}$ the momenta of the reduced particle of the complete system in the entry and exit channels, respectively, with $Z_{p}$ being the projectile charge and $Z_{T}^{*}$ an effective target charge. $N^{*}(a)$ refers to the conjugate of the quantity $N(a)=\exp (\pi a / 2) \Gamma(1-i a)$. Besides, $\phi_{\alpha}(\boldsymbol{x})$ and $\phi_{\beta}(\boldsymbol{x})$ describe the bound electron wave function in the entry channel and a plane wave described from the target framework in the exit channel, respectively. Note that the asymptotic boundary conditions associated with the projectile-active electron interaction are preserved, but $\varphi_{\alpha}^{+}$presents a one-target center character. In the exit channel [see Eq. (4)], an asymptotic version of this interaction is also considered (depending on $R$ ), which will be valid under the dynamic condition $\boldsymbol{k} \ll \boldsymbol{v}(\boldsymbol{x} \ll \boldsymbol{R})$. So, in the CB1 approximation for ionization, correct boundary conditions are only satisfied in this restricted coordinate space region. Thus, $\varphi_{\beta}^{-}$presents also a one-target center character. It must also be mentioned that the application of the active electron Schrödinger equation on the wave function in Eq. (3) results in

$$
\left(H_{\alpha}-E_{\alpha}\right) \phi_{\alpha}^{+}=V_{\alpha} \phi_{\alpha}^{+}
$$

with the perturbative potential given by

$$
V_{\alpha}=-\frac{Z_{p}}{s}+\frac{Z_{p}}{R},
$$

where $s$ is the position vector of the active electron with respect to the projectile. Finally, note that in the present quantum-mechanical calculations, the effective target charge $Z_{T}^{*}$ is taken as $Z_{T}^{*}=\sqrt{-2 n_{\alpha}^{2} \varepsilon_{\alpha}}$, where $n_{\alpha}$ refers to the principal quantum number of each atomic orbital component used in each molecular orbital expansion whereas the active electron orbital energy $\varepsilon_{\alpha}$ is related to the ionization energies $B_{j}$ of the occupied molecular orbitals by $\varepsilon_{\alpha}=-B_{j}$. Each molecular orbital is thus described by using a basis of effective atomic ones. 


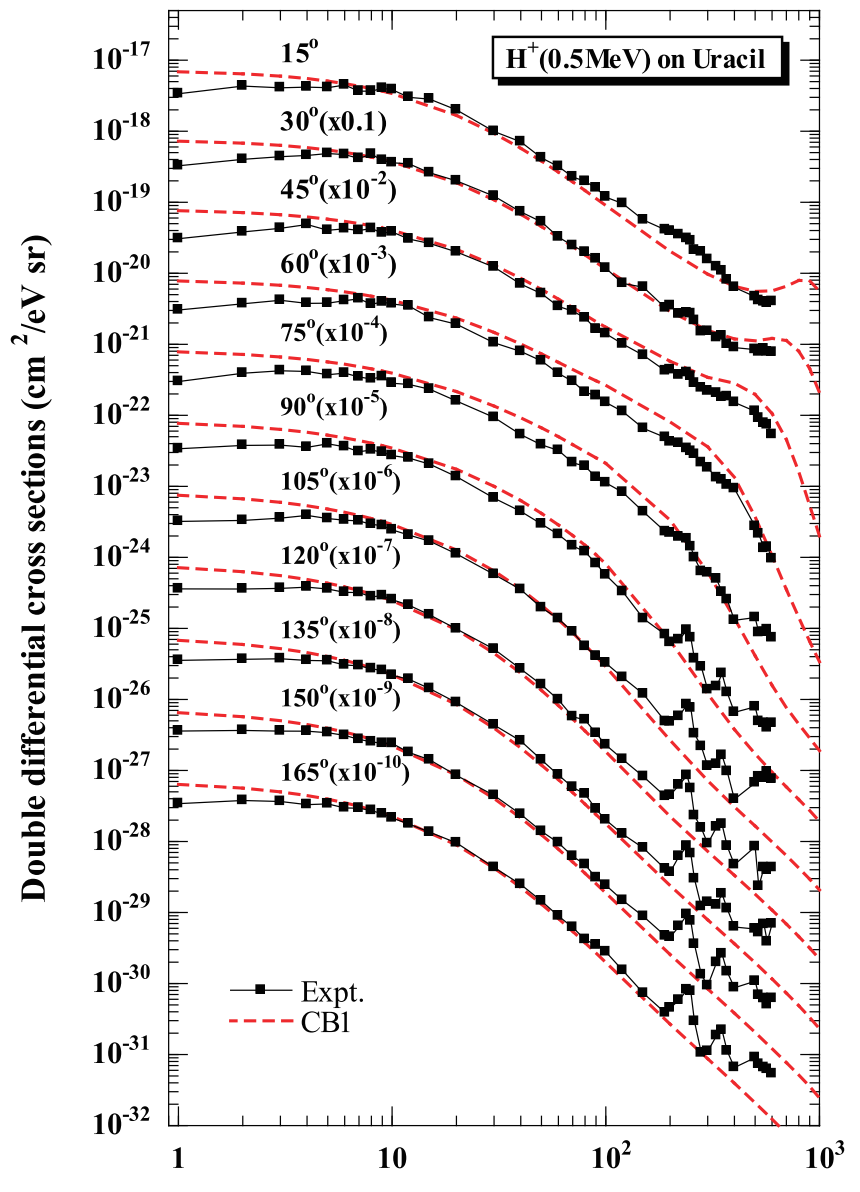

Ejected electron energy $(\mathrm{eV})$

FIG. 1. (Color online) Double differential cross sections (DDCS) for electron emission from uracil impacted by $0.5 \mathrm{MeV}$ protons. DDCS are plotted by multiplying scaling factors as shown in parentheses. Calculated values with CB1 are depicted by red dashed lines.

\section{B. Classical calculation}

Analytical formulas for SDCS and TDCS were derived by Stolterfoht et al. [4]. They obtained the following expressions within the framework of the classical binary Rutherford collision. The classical SDCS is given by

$$
\begin{aligned}
\sigma_{c l}(\epsilon) & =\sum_{j} \frac{4 \pi a_{0}^{2} Z_{p}^{2} E_{R}^{2}}{T\left(c_{j} B_{j}+\epsilon\right)^{2}}, \\
\text { with } \quad c_{j} & =\left[\ln \left(\frac{2 T}{B_{j}}\right)\right]^{-1 / 2},
\end{aligned}
$$

where $a_{0}$ is the Bohr radius $\left(0.53 \times 10^{-8} \mathrm{~cm}\right), E_{R}$ the Rydberg energy $(13.6 \mathrm{eV})$, and $T=m v^{2} / 2$ the kinetic energy of the electron of mass $m$ and the velocity $v$ same as the incident proton. The TICS obtained by integrating the above equation over $\epsilon$ is

$$
\sigma_{t, c l}=\frac{4 \pi a_{0}^{2} Z_{p}^{2}}{T} \sum_{j} \frac{E_{R}^{2}}{B_{j}} \sqrt{\ln \left(\frac{2 T}{B_{j}}\right)} .
$$

The calculation was made over all subshell electrons including $1 s$ shells.

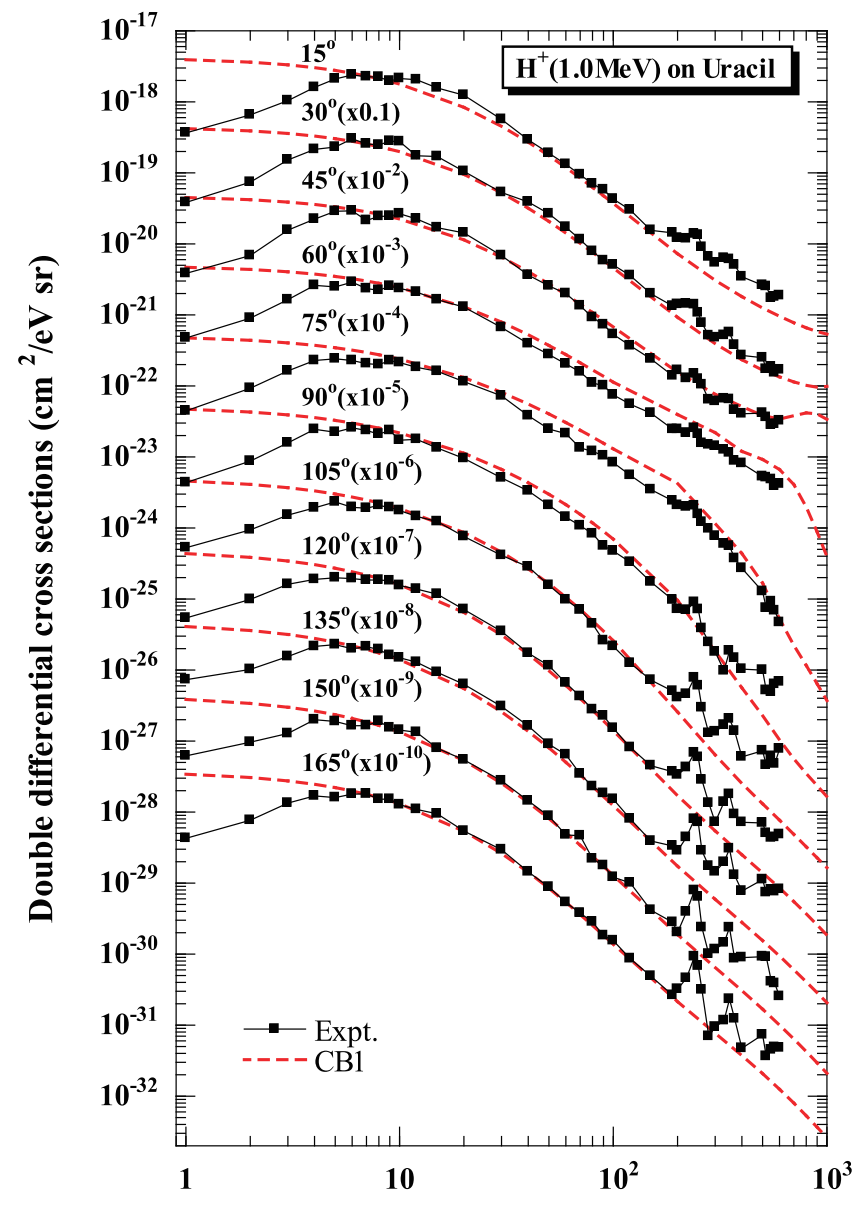

Ejected electron energy $(\mathrm{eV})$

FIG. 2. (Color online) The same as Fig. 1 but for $1.0 \mathrm{MeV}$ proton impact.

\section{RESULTS AND DISCUSSION}

\section{Ionization cross sections}

Figures 1,2, and 3 show DDCS obtained for gas phase uracil molecules impacted by $0.5,1.0$, and $2.0 \mathrm{MeV}$, respectively. All these numerical data are presented in the Supplemental Material [32]. The uncertainties in the absolute value of the measured DDCS are 11-15\%. The theoretical values by the CB1 calculation are depicted by red dashed lines in these figures.

One can see that the experimental and theoretical values coincide fairly well at all emission angles besides a low-energy region of $\epsilon \leqslant 7 \mathrm{eV}$. Peaks observed at about 250, 350, and $450 \mathrm{eV}$ are attributed to $K-L L$ Auger electrons ejected from carbon, nitrogen, and oxygen, respectively. The classical binary encounter peaks expected at $\epsilon=4 T \cos ^{2} \theta$ are well reproduced by $\mathrm{CB} 1$ calculation at small $\theta$ 's.

Experimental cross sections increase with decreasing electron energy down to about $7 \mathrm{eV}$ and then decrease at lower energies, while theoretical values keep increasing in this energy range. As reported in Ref. I this "hump" behavior was also observed for adenine but not for Ar target for which cross sections are nearly flat being in agreement with the data of Toburen et al. tabulated in [33]. It is interesting to note that the hump becomes sharper as the incident proton energy 


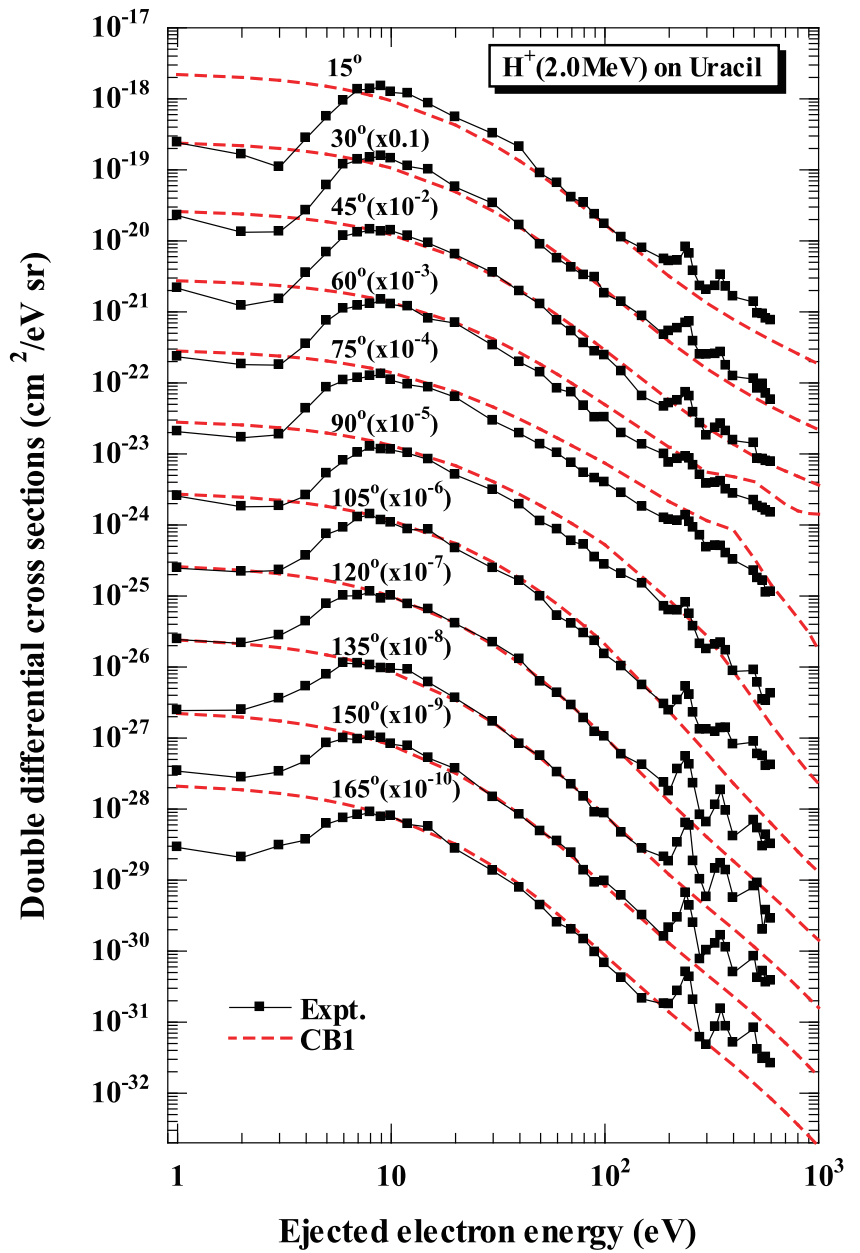

FIG. 3. (Color online) The same as Fig. 1 but for $2.0 \mathrm{MeV}$ proton impact.

increases; e.g., compare the data between 0.5 and $2 \mathrm{MeV}$. Although the origin causing this hump structure is not clear at present, it should be noted that the DDCS spectra were well reproduced in several repeated measurements under different conditions. Namely, measurements were done at different target pressures by changing the oven temperature and by changing the height of the oven. Effects due to stray magnetic fields from the heater are ruled out because measurements were also done under a power-off condition of the oven heaters at desired temperatures. A possible explanation is the absorption of low-energy electrons by collision with gas molecules in the chamber. If so, however, this absorption effect does not explain why it is large at $2.0-\mathrm{MeV}$ impact compared to lower-energy impacts. In conclusion, therefore, it remains as an open question.

Figure 4 shows a comparison of experimental SDCS with theoretical results of $\mathrm{CB} 1$ and classical Rutherford cross sections from Eq. (7). Experimental results are in excellently good agreement with CB1 above $10 \mathrm{eV}$. As mentioned above, the discrepancy at energies below $7 \mathrm{eV}$ increases with increasing proton energy. However, it should be kept in mind that discrepancies in this region do not contribute significantly to the values of TICS obtained by integrating the SDCS spectra. We found the difference of TICS obtained by using flat cross

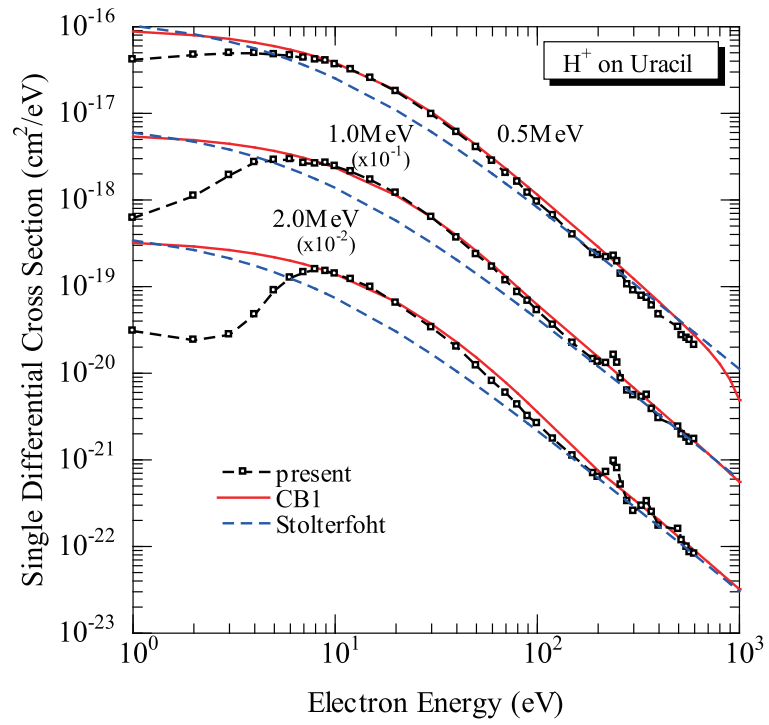

FIG. 4. (Color online) Single differential cross sections (SDCS) of uracil as a function of the incident proton energy. Experimental results (squares) are compared to theoretical calculations: CB1 (red lines) and classical Rutherford cross sections (blue dashed lines) from Eq. (7).

sections instead of measured values is only $5 \%$ at $2 \mathrm{MeV}$ and less than $1 \%$ at $0.5 \mathrm{MeV}$.

It is noteworthy that the Eq. (7) gives nearly the same order of magnitude as the quantum-mechanical calculation except the electron energy region from a few eV to $100 \mathrm{eV}$. Here, we point out that Eq. (7) approaches the $B_{j}$-independent cross section at higher electron energies of $\epsilon \gg B_{j}$. Under this condition, the SDCS becomes proportional to the

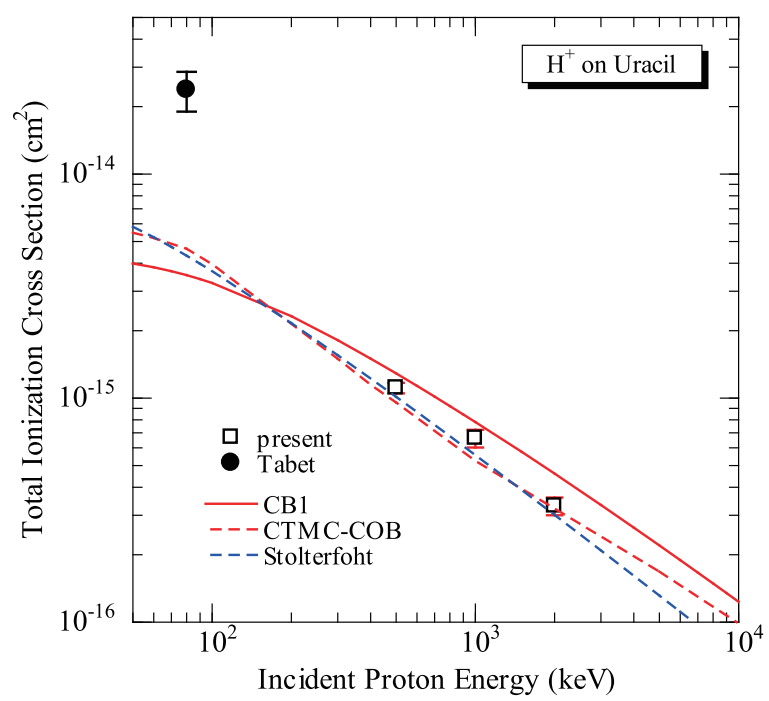

FIG. 5. (Color online) Total ionization cross sections (TICS) of uracil as a function of the incident proton energy. Present experimental results are depicted by open squares. Calculated values with $\mathrm{CB} 1$ are shown by a red solid line. Classical cross sections with Eq. (9) are shown by a blue dashed line. Theoretical results of CTMC-COB are also shown by a red dashed line [24]. Experimental value at $80 \mathrm{keV}$ (closed circle) is taken from [20]. 


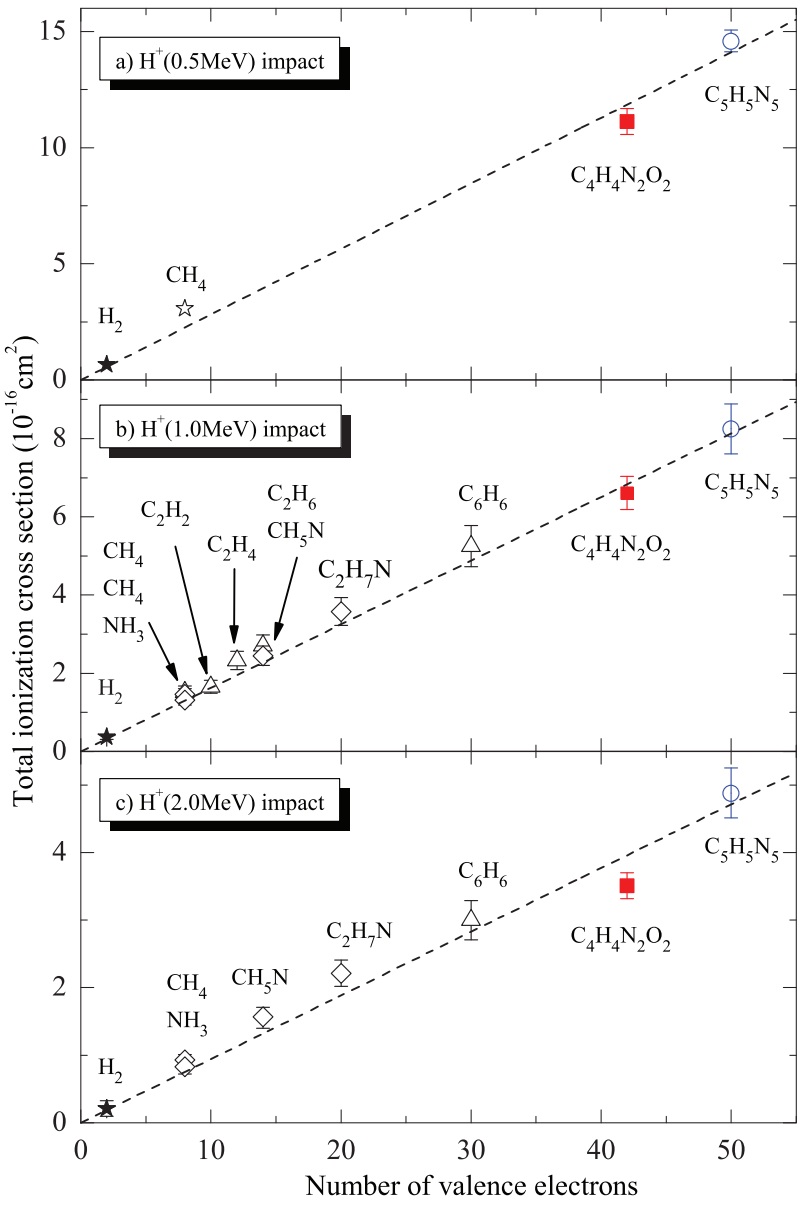

FIG. 6. (Color online) TICS plotted versus $n_{v}$ for various molecules obtained for $0.5,1.0$, and $2.0 \mathrm{MeV}$ proton impacts. Data are taken from Ref. I for adenine, from [14] for $\mathrm{H}_{2}$, and from $[9,10]$ for other molecules.

effective number of target electrons. The SDCS per single electron is thus given by $\sigma_{c l}(\epsilon)\left[\mathrm{cm}^{2} / \mathrm{eV} /\right.$ electron $]=6.53 \times$ $10^{-14} /\left(T \epsilon^{2}\right)$. At $1.0 \mathrm{MeV}$ impact $(T=544.7 \mathrm{eV})$, it gives $1.2 \times 10^{-22} \mathrm{~cm}^{2} / \mathrm{eV} /$ electron at $\epsilon=1000 \mathrm{eV}$. On the other hand, the value of SDCS shown in Fig. 4 at this $\epsilon$ is about $5.5 \times 10^{-21}$. Hence, the ratio between these two values is 45.8 , which is a good measure of the effective number of target electrons contributing to ionization as discussed in [13,34]. Similarly, these ratios are 41.8 and 50 for 0.5 and $2.0 \mathrm{MeV}$, respectively. Suffice it to say, these values are very close to 42 , the total number of valence shell electrons of uracil rather than the actual total number of electrons of 58. This is because the binding energies of valence electrons lie between $B_{1}=9.5 \mathrm{eV}$ and $B_{21}=37.9 \mathrm{eV}$, which are one order of magnitude smaller than the $1 s$ electrons.
Figure 5 shows TICS for uracil molecules as a function of the incident proton energy. Together with the present theoretical results of CB1 and Eq. (9), the CTMC-COB results of Lekadir et al. [24] are also shown. Our experimental results are in fairly good agreement with all these theoretical curves within about $20 \%$. An experimental value plotted at $80 \mathrm{keV}$, obtained by Tabet et al. [20] from time-of-flight measurements of fragment ions, is obviously too large compared to other data.

Finally, the scaling property of TICS is described briefly. The scaling property of proton-impact ionization cross sections has been considerably investigated by Toburen et al. for various polyatomic molecules [9-11]. They found that cross sections are well scaled within $\pm 15 \%$ in terms of the number of weakly bound target electrons $n_{v}$ of each molecule. As shown in [10], the total ionization cross sections $\sigma_{t}$ at a given proton energy are well proportional to $n_{v}$, showing straight lines connecting experimental values of molecules of up to benzen $\left(n_{v}=30\right)$. We found in Ref. I that these scaling lines can be extended to adenine $\left(n_{v}=50\right)$. Here, the cross sections for uracil $\left(n_{v}=42\right)$ are also found to lie on these lines reasonably as demonstrated in Fig. 6. The formula of best-fit lines in units of $10^{-16} \mathrm{~cm}^{2}$ are obtained as $\sigma_{t}=0.282 n_{v}$ $(0.5 \mathrm{MeV}), 0.163 n_{v}(1.0 \mathrm{MeV})$ and $0.094 n_{v}(2.0 \mathrm{MeV})$. The validity of this scalability is simply because the velocities of weakly bound electrons of $\mathrm{C}, \mathrm{N}$, and $\mathrm{O}$ are much slower than the incident protons and ionization cross sections for these electrons do not differ greatly.

\section{CONCLUSION}

Experimental and theoretical investigations have been performed for the ionization of gas phase uracil molecules bombarded by $0.5,1.0$, and $2.0 \mathrm{MeV}$ protons. The experimental values of DDCS, SDCS, and TICS are in fairly good agreement with theoretical calculations of both quantum-mechanical and classical methods. It is noted that the simple analytical formula proposed by Stolterfoht $e t$ al. [4] gives also equivalent cross sections. Investigation should be extended more to other polyatomic molecules as the origin of discrepancies between experiment and theory remains unknown.

\section{ACKNOWLEDGMENTS}

This work was supported by a Grant-in-Aid for Scientific Research (B), Grant No. 24340096, from the Japan Society for the Promotion of Science. Also, this work has been developed as part of the activities planned in the Programme de Coopération PICS 5921 (THEOS) as well as the PEPS-PTI 2013 (ARISTOTE) of the Centre National de la Recherche Scientifique, the project PICT 2145 of the Agencia Nacional de Promoción Científica y Tecnológica and the Projects No. PIP 1026 and No. PIP 0033 from CONICET.
[1] M. E. Rudd, Y. Kim, D. H. Madison, and J. W. Gallagher, Rev. Mod. Phys. 57, 965 (1985).

[2] M. E. Rudd, Y. Kim, D. H. Madison, and T. J. Gay, Rev. Mod. Phys. 64, 441 (1992).
[3] M. E. Rudd, Y. Kim, T. Märk, J. Schou, N. Stolterfoht, L. H. Toburen, H. Bichsel, R. D. Dubios, H. O. Wyckoff, W. R. Ney, H. G. Ebert, and O. Linton, ICRU Report No. 55, Oxford, 1995 (unpublished). 
[4] N. Stolterfoht, R. D. DuBois, and R. D. Rivarola, Electron Emission in Heavy-Ion Atom Collision (Springer, Berlin, 1997).

[5] F. Gobet, B. Farizon, M. Farizon, M. J. Gaillard, M. Carré, M. Lezius, P. Scheier, and T. D. Märk, Phys. Rev. Lett. 86, 3751 (2001).

[6] S. Martin, L. Chen, A. Salmoun, B. Li, J. Bernard, and R. Brédy, Phys. Rev. A 77, 043201 (2008).

[7] M. Schulz and D. H. Madison, Int. J. Mod. Phys. B 21, 3649 (2006).

[8] M. Schulz, B. Najjari, A. B. Voitkiv, K. Schneider, X. Wang, A. C. Laforge, R. Hubele, J. Goullon, N. Ferreira, A. Kelkar, M. Grieser, R. Moshammer, J. Ullrich, and D. Fischer, Phys. Rev. A 88, 022704 (2013).

[9] W. E. Wilson and L. H. Toburen, Phys. Rev. A 11, 1303 (1975).

[10] D. J. Lynch, L. H. Toburen, and W. E. Wilson, J. Chem. Phys. 64, 2616 (1976).

[11] L. H. Toburen, W. E. Wilson, and L. E. Porter, J. Chem. Phys. 67, 4212 (1977).

[12] L. H. Toburen and W. E. Wilson, J. Chem. Phys. 66, 5202 (1977).

[13] L. H. Toburen, S. T. Manson, and Y. K. Kim, Phys. Rev. A 17, 148 (1978).

[14] M. E. Rudd, R. D. DuBois, L. H. Toburen, C. A. Ratcliffe, and T. V. Goffe, Phys. Rev. A 28, 3244 (1983).

[15] M. E. Rudd, T. V. Goffe, R. D. DuBois, and L. H. Toburen, Phys. Rev. A 31, 492 (1985).

[16] M. E. Rudd, A. Itoh, and T. V. Goffe, Phys. Rev. A 32, 2499 (1985).

[17] M. A. Bolorizadeh and M. E. Rudd, Phys. Rev. A 33, 882 (1986).

[18] F. Gobet, S. Eden, B. Coupier, J. Tabet, B. Farizon, M. Farizon, M. J. Gaillard, M. Carré, S. Ouaskit, T. D. Märk, and P. Scheier, Phys. Rev. A 70, 062716 (2004).
[19] D. Ohsawa, Y. Sato, Y. Okada, V. P. Shevelko, and F. Soga, Phys. Rev. A 72, 062710 (2005).

[20] J. Tabet, S. Eden, S. Feil, H. Abdoul-Carime, B. Farizon, M. Farizon, S. Ouaskit, and T. D. Märk, Phys. Rev. A 82, 022703 (2010).

[21] P. Moretto-Capelle and A. Le Padellec, Phys. Rev. A 74, 062705 (2006).

[22] A. N. Agnihotri, S. Kasthurirangan, S. Nandi, A. Kumar, M. E. Galassi, R. D. Rivarola, O. Fojón, C. Champion, J. Hanssen, H. Lekadir, P. F. Weck, and L. C. Tribedi, Phys. Rev. A 85, 032711 (2012).

[23] A. Abbas, C. Champion, B. Zarour, B. Lasri, and J. Hanssen, Phys. Med. Biol. 53, N41 (2008).

[24] H. Lekadir, I. Abbas, C. Champion, O. Fojón, R. D. Rivarola, and J. Hanssen, Phys. Rev. A 79, 062710 (2009).

[25] C. Champion, H. Lekadir, M. E. Galassi, O. Fojon, R. D. Rivarola, and J. Hanssen, Phys. Med. Biol. 55, 6053 (2010).

[26] L. Sanche, Mass Spectro. Rev. 21, 349 (2002).

[27] L. Sanche, Eur. Phys. J. D 35, 367 (2005).

[28] S. Lehnert, Biomolecular Action of Ionizing Radiation (Taylor \& Francis, London, 2008).

[29] Y. Iriki, Y. Kikuchi, M. Imai, and A. Itoh, Phys. Rev. A 84, 032704 (2011); 84, 052719 (2011).

[30] N. S. Hush and A. S. Cheung, Chem. Phys. Lett. 34, 11 (1975).

[31] M. E. Galassi, C. Champion, P. F. Weck, R. D. Rivarola, O. Fojón, and J. Hanssen, Phys. Med. Biol. 57, 2081 (2012).

[32] See Supplemental Material at http://link.aps.org/supplemental/ 10.1103/PhysRevA.88.052711 for numeric data on double differential cross sections.

[33] M. E. Rudd, L. H. Toburen, and N. Stolterfoht, At. Data Nucl. Data Tables 23, 405 (1979).

[34] Y.-K. Kim, Radiat. Res. 61, 21 (1975); 64, 205 (1975). 